\title{
A Grande Elevação Eustática do Mioceno e Sua Influência na Origem do Grupo Barreiras
}

\author{
Mitsuru Arai (arai@petrobras.com.br) \\ PETROBRAS/CENPES/PDEXP/BPA \\ Ilha do Fundão, Quadra 7 - Cidade Universitária, CEP 21941-598, Rio de Janeiro, RJ, BRA \\ Recebido em 31 de janeiro de 2006; aceito em 15 de junho de 2006
}

Palavras-chave: Grupo Barreiras, Mioceno, Plioceno, Brasil.

\section{RESUMO}

O Grupo Barreiras é uma unidade que ocorre ao longo da faixa costeira do Brasil, desde o Estado do Amapá até o Rio de Janeiro, caracterizando-se pela ocorrência quase contínua e pela regularidade geomorfológica. Sua origem tem sido motivo de muitas discussões. Tradicionalmente, a unidade vinha sendo considerada como de origem continental, mas trabalhos recentes vêm mostrando evidências irrefutáveis de influência marinha, tanto de natureza paleontológica, como sedimentológica. A datação palinológica e sua correlação com as unidades litoestratigráficas coevas das partes submersas das bacias da margem continental brasileira e das outras partes do planeta permitiram um estudo integrado sob a luz da Estratigrafia de Seqüências. Essa análise estratigráfica integrada permitiu relacionar a origem do Grupo Barreiras com a elevação eustática global que teve seu máximo na parte média do Mioceno, mais precisamente no intervalo do Burdigaliano ao Serravaliano (12 - $20 \mathrm{Ma})$. A sedimentação do Barreiras lato sensu sofreu uma interrupção no Tortoniano (início do Neomioceno), quando houve um rebaixamento eustático global que ocasionou um extenso evento erosivo nas áreas emersas e a formação de cunhas fortemente progradantes na porção submersa das bacias submersas (offshore). Com a retomada da subida eustática no Plioceno (Zancleano, 4 - $5 \mathrm{Ma}$ ), depositou-se o segundo ciclo (Barreiras Superior). A erosão e o retrabalhamento do Grupo Barreiras no Quaternário devem ter sido responsáveis, em parte, pela atual configuração da plataforma continental.

Keywords: Barreiras Group, Miocene, Pliocene, Brazil.

\begin{abstract}
The Barreiras Group is a lithostratigraphic unit, which crops out extensively along the Brazilian coast, from the state of Amapá (North Brazil) to the state of Rio de Janeiro (Southeast Brazil). It is characterized by its continuous occurrence and geomorphological regularity. Its origin has been the subject of much discussion and controversy. Traditionally, it has been considered as continental, but recent studies have shown an irrefutable marine influence, as indicated by paleontological and sedimentological evidence. The palynological dating and correlation of the Barreiras Group with coeval lithostratigraphic units in offshore basins of the Brazilian continental margin and elsewhere in the world permit new interpretations in the light of sequence stratigraphy concepts. This integrated analysis permits correlation of the origin of the Barreiras Group with the Miocene global eustatic rise, which reached its maximum from the Burdigalian to the Serravallian (12 - 20 Ma). Deposition of the Barreiras sensu lato was interrupted in the Tortonian (early Late Miocene), when a global eustatic fall exposed and eroded part of the Barreiras and led to formation of prograding wedges in the offshore portion of continental margin basins. Renewed eustatic rise in the Pliocene (Zanclean, $4-5 \mathrm{Ma}$ ) initiated a second depositional phase, represented by the upper part of the Barreiras Formation. Erosion and reworking of the Barreiras Group during the Quaternary have contributed to the present configuration of the continental shelf.
\end{abstract}




\section{INTRODUÇÃO}

O Grupo Barreiras (ou a Formação Barreiras para alguns) chamou atenção dos navegadores portugueses que chegaram à costa brasileira em 1500, por apresentar uma geomorfologia muito peculiar. Essa feição - "barreiras" ocorre de modo consistente ao longo do litoral brasileiro, desde o Estado do Amapá até o Estado do Rio de Janeiro. Sua regularidade no modo de ocorrência e na característica litológica é incompatível com a origem continental apregoada tradicionalmente (e.g., Oliveira e Leonardos, 1940; Mendes e Petri, 1971; Bigarella, 1975), o que constitui um mistério. Alguns autores já haviam revelado a presença de fósseis marinhos (Bassler, in Oliveira e Leonardos, 1943, p. 680; Beurlen, 1964) e de restos de vegetação costeira (Salim et al., 1975) em seus estratos. Entretanto, estudos sistemáticos revelando irrefutavelmente o caráter marinho do Barreiras começaram a surgir somente a partir dos anos 80 no Norte do Brasil (Arai et al., 1988, 1994; Rossetti, Truckenbrodt e Góes, 1989; Rossetti, Góes e Truckenbrodt, 1990; Costa et al., 1993; Truckenbrodt et al., 1994; Leite, 1997; Leite et al., 1997; Arai, 1997). A região Nordeste ainda não revelou evidências contundentes de caráter marinho, limitando-se a oferecer apenas fácies sugestivas de ambiente transicional (Alheiros et al., 1988; Alheiros e Lima Filho, 1991). Recentemente, o caráter marinho foi confirmado também para a ocorrência do sul do Estado da Bahia (Arai e Novais, 2006). Devido ao seu pobre conteúdo fossilífero, a idade do Grupo Barreiras tem sido motivo de muita controvérsia. $\mathrm{Na}$ literatura mais antiga, a idade variava de Oligoceno Mioceno a Plioceno. Mais recentemente, estudos palinológicos efetuados por Arai et al. $(1988,1994)$ têm posicionado a parte inferior do Barreiras no Mioceno inferior a médio, além de admitir a interdigitação desta com a Formação Pirabas, na região Norte do Brasil (Pará e Maranhão). A julgar pela datação efetuada por meio de foraminíferos (Petri, 1972), a Formação Sabiá, no Recôncavo Baiano, é correlacionável com a Pirabas e, portanto, pode ser colocada no mesmo contexto.

\section{ESTUDO INTEGRADO}

$\mathrm{O}$ arcabouço estratigráfico geral, integrando as subunidades do Grupo Barreiras e as unidades coevas das partes submersas das bacias da margem continental, foi obtido graças à disponibilidade de grande número de datações efetuadas por meio de microfósseis - os foraminíferos, os palinomorfos e principalmente os nanofósseis calcários. Um dos horizontes de correlação inter-regional é a Discordância Tortoniana (Shimabukuro e Arai, 2000) que foi produzida por um evento erosivo ocor- rido no Tortoniano (11,6 - 7,2 Ma). O evento foi ocasionado pela maior queda eustática do Neógeno, cuja causa está na formação da calota glacial da Antártida há cerca de 10 milhões de anos (Burckle, Keigwin e Opdyke, 1982; Viana, Castro e Kowsmann, 1990). Nas bacias da margem continental, a Discordância Tortoniana é identificada em seções sísmicas, onde é reconhecida como "Marco Cinza" (Viana, Castro e Kowsmann, 1990). A idade tortoniana do hiato é atribuída a partir da ausência quase sistemática das biozonas de nanofósseis Discoaster hamatus (N-630) e D. neorectus (N-635). Por representar depósitos de mar baixo (lowstand tract), nas bacias submersas da margem continental, os estratos tortonianos e os pós-tortonianos imediatamente sobrejacentes exibem, quando presentes, abundantes fósseis pré-tortonianos retrabalhados.

O estudo integrado do Grupo Barreiras juntamente com as unidades coevas das bacias submersas da margem continental brasileira permitiu elaborar um arcabouço sob a luz da Estratigrafia de Sequiências (Shimabukuro e Arai, 1999, 2000, 2001; Arai e Shimabukuro, 2003).

\section{CENÁRIO GEOLÓGICO DO GRUPO BARREIRAS}

Antes do evento erosivo do Tortoniano, o cenário na margem continental brasileira era dominado pela grande transgressão do intervalo Aquitaniano - Serravaliano (Eomioceno - Mesomioceno) que foi responsável pela acumulação de uma grande quantidade de sedimentos nas áreas hoje emersas do continente (Figura 1). Conhecem-se vários relictos de depósitos transgressivos e de mar alto (highstand tract) que se manifestam em forma de unidades litoestratigráficas quase contínuas como o Grupo Barreiras, ou em forma mais ou menos isolada como as formações Pirabas e Sabiá, entre outras. Estes depósitos foram sedimentados em áreas extensas - sobretudo na faixa costeira que vai atualmente da Foz do Amazonas ao estado do Rio de Janeiro -, onde a ausência de topografia elevada teria propiciado o avanço do onlap para continente adentro. Todavia, devido à erosão sofrida no Tortoniano, sua ocorrência atual é restrita. No Zancleano (Plioceno), de acordo com as curvas eustáticas propostas na literatura (Haq, Hardenbol e Vail, 1987), teria ocorrido uma nova transgressão (Figura 1), e muitas das áreas erodidas receberam uma nova cobertura que viria a constituir o "Barreiras Superior". Mais para o interior - por exemplo, no Alto Amazonas, no Alto Xingu e no Planalto de Borborema - o horizonte que representaria a Discordância Tortoniana se encontra exumado, ou se encontra ampliado em forma de hiato entre o Pré-Tortoniano e o Quaternário, pois estas áreas não foram atingidas pela transgressão do Zancleano. 

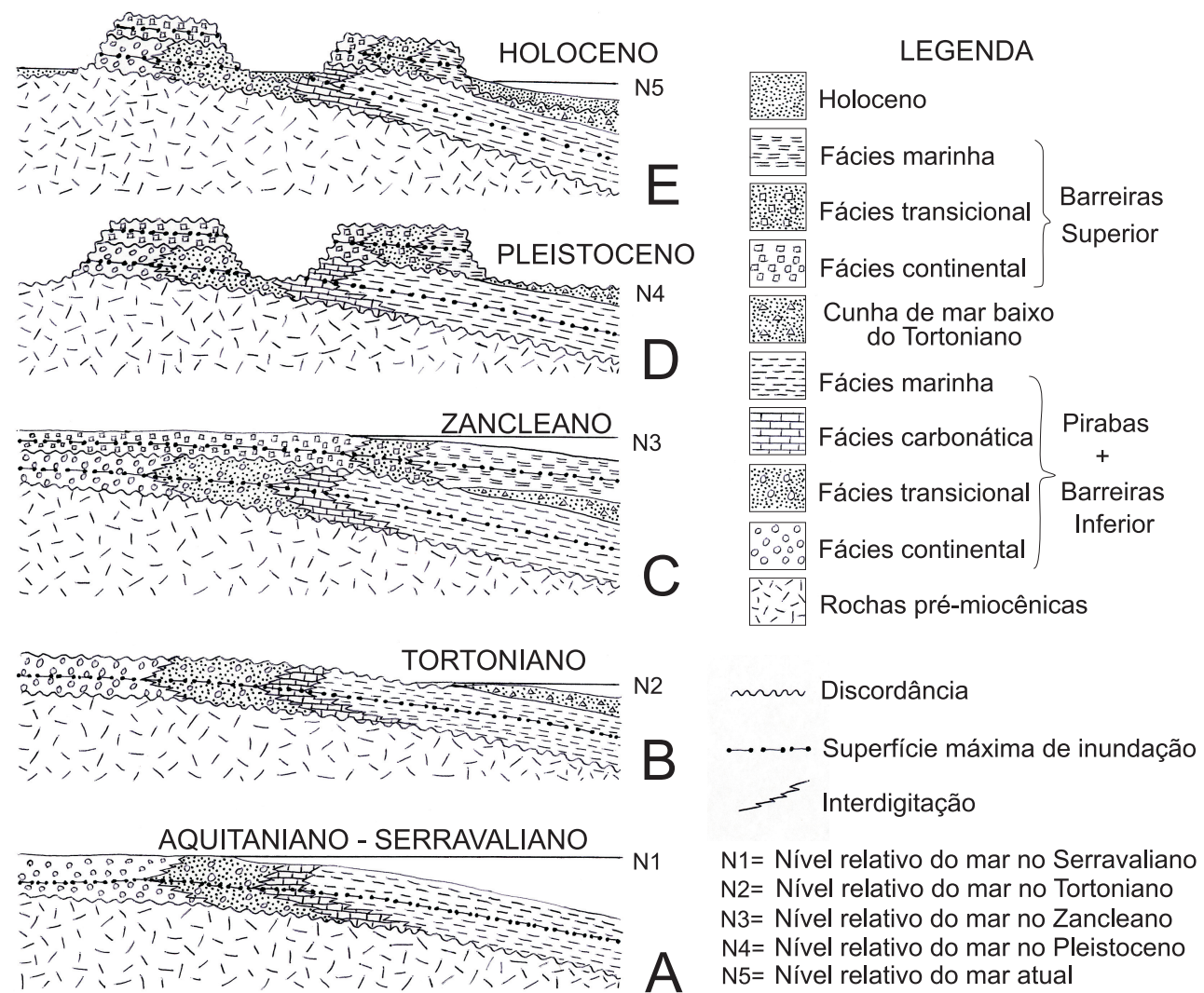

$\mathrm{N} 1=$ Nível relativo do mar no Serravaliano N2= Nível relativo do mar no Tortoniano N3= Nível relativo do mar no Zancleano N4= Nível relativo do mar no Pleistoceno $\mathrm{N} 5=$ Nível relativo do mar atual

Figura 1. Esquema de evolução do Grupo Barreiras lato sensu na costa do Norte do Brasil. A. Sistema transgressivo do intervalo Aquitaniano - Burdigaliano e sistema de mar alto do intervalo Langhiano - Serravaliano. B. Sistema de mar baixo do Tortoniano; formação da Discordância Tortoniana. C. Sistemas transgressivo e de mar alto do Zancleano (Plioceno). D. Fase erosiva do máximo da regressão do Pleistoceno. E. Erosão e retrabalhamento dos sedimentos do Grupo Barreiras no Holoceno; sedimentação quaternária em forma de depósitos de praia e aluvião; as ocorrências residuais do Grupo Barreiras formam falésias e tabuleiros costeiros. Sem escala.

\section{CENÁRIO EM ESCALA CONTINENTAL (AMÉRICA DO SUL)}

A rigor, a influência da transgressão miocênica não se limitou à área costeira do Brasil. No Burdigaliano (Eomioceno tardio) extensas áreas continentais teriam sido cobertas por um mar epicontinental. No caso da América do Sul, alguns autores estimaram que cerca de um terço do continente teria sido inundado pelo mar alto nessa época (e.g., Webb, 1995). Embora a idéia de ingressão marinha exista desde que fora preconizada por Ihering (1927, apud Boltovskoy, 1991) para o Neocretáceo e retomada por outros autores para o Terciário, foram Räsänen et al. (1995) que apresentaram provas substanciais testáveis. Essa ingressão marinha, segundo o modelo proposto, teria ocupado a faixa que vai do norte da Argentina ao Peru, bordejando a Cordilheira dos Andes. No
Peru, o mar epicontinental, conectado com o Pacífico, teria tido mais duas ramificações através de dois corredores: um conectando ao norte, com o Atlântico Caribenho, e outro a leste, ligando-se ao que é hoje a foz do Amazonas. Neste processo, extensos depósitos sedimentares epicontinentais de mar alto teriam sido depositados, constituindo o que poderia ser chamado de "Barreiras lato sensu" com diversas denominações locais (e.g., formações Pebas, na Bacia Marañon; Ipururo, na Bacia Huallaga; e Solimões, nas bacias de Solimões e Acre).

\section{EVENTOS PÓS-DEPOSICIONAIS}

Após a deposição do "Barreiras Superior", processos erosivos passaram a ser dominantes em função da conjunção de dois fatores: 
1. a queda eustática pós-zancleana;

2. o soerguimento epirogenético pós-pliocênico.

O soerguimento associado ao arqueamento crustal é particularmente notável no Nordeste do Brasil, onde as ocorrências residuais do Grupo Barreiras e unidades correlatas formam tabuleiros mais ou menos isolados que ocorrem em cotas topográficas cada vez maiores rumo ao interior. O topo dos tabuleiros e a cimeira das chapadas cretáceas estão alinhados segundo uma curva ascendente que parte da cota próxima ao nível do mar no litoral e que ascende a altitudes de centenas de metros no seio do Planalto da Borborema, o que sugere que o mesmo soerguimento que levantou as chapadas foi responsável pela elevação dos tabuleiros.

\section{COMENTÁRIOS ADICIONAIS}

Com relação ao Barreiras, existe ainda um paradigma tradicional fortemente enraizado, e, em função disso, muitos estudos impecáveis sob o ponto de vista metodológico continuam realizando interpretações inadequadas. Provavelmente, muitas das fácies descritas como "sistema fluvial meandrante" (Araújo et al., 2005), "ambiente fluvial entrelaçado distal" e "planície de inundação fluvial" (Morais et al., 2005) seriam associadas a ambiente costeiro transicional. O autor deste artigo não estudou os afloramentos descritos por aqueles autores, mas, dentro da previsibilidade que o presente modelo oferece, seria grande a possibilidade de encontrar fácies com influência significativa de maré naquelas paragens.

A icnologia - estudo de icnofósseis, vulgarmente conhecidos como bioturbações - desponta como uma ferramenta muito útil na caracterização paleoambiental do Barreiras. Alguns trabalhos pioneiros já foram realizados nos afloramentos das regiões Norte e Meio-Norte (Netto e Rossetti, 2003; Rossetti, 2005a, 2005c). É desejável que estudos similares sejam realizados sistematicamente em afloramentos, no sentido de caracterizar o padrão de sucessão icnofaciológica em outras regiões. Convém salientar que uma ocorrência isolada de número reduzido de icnofósseis não constitui prova para definição de paleoambientes, mas uma sucessão coerentemente cadenciada de determinadas icnofácies pode indicar com precisão o tipo de paleoambiente e a mudança ocorrida nele.

Novas tecnologias acenam com contribuições inéditas. Como exemplo, podem ser mencionadas a datação radiométrica de eventos de intemperismo e lateritização (palestra proferida por Paulo Vasconcelos em 10/10/2005, no Simpósio "Significado Geológico da Formação Barreiras" realizado no âmbito do X Congresso da ABEQUA; Vasconcelos, 2005) e a aplicação do Radar de Penetração Subterrânea (Santos et al., 2005).
Em observações de campo, baseadas somente em critérios litológicos e sedimentológicos, persiste ainda a dificuldade para correlacionar as discordâncias observadas no litoral do Espírito Santo com aquelas descritas no Norte e Meio-Norte (Rossetti, 2005b). Certamente, análises de sucessão icnofaciológica e outras técnicas novas a serem aplicadas poderão auxiliar na correlação.

A discussão acerca da hierarquia litoestratigráfica do Barreiras - se a unidade deve ser considerada "Formação" ou "Grupo" - continua em pauta, mas a julgar da existência de diversas formações já definidas por diversos autores, o mais sensato seria a adoção de "Grupo". Além disso, se a Discordância Tortoniana separa a Unidade Barreiras em pelo menos duas sequiências bem distintas, é preferível a adoção de "Grupo". Esta posição tem respaldo da Comissão Internacional de Estratigrafia (ICS, 2005) que recomenda que as discordâncias de expressão regional sejam levadas em consideração, para separar unidades litoestratigráficas. Entre os trabalhos apresentados no simpósio, o de Ferraz e Valadão (2005) apreciou esta questão e deu parecer favorável ao uso do termo Grupo Barreiras.

\section{CONCLUSÃO}

O Sistema Pirabas/Barreiras Inferior foi depositado durante a subida eustática ocorrida no intervalo Aquitaniano - Serravaliano (Eomioceno a Mesomioceno).

A unidade Barreiras Superior foi depositado no Plioceno, existindo, entre esta e a unidade Barreiras Inferior, a Discordância Tortoniana.

O soerguimento epirogenético, ocorrido subseqüentemente à queda eustática, foi responsável pela atual configuração topográfica do Grupo Barreiras.

A erosão e o retrabalhamento, ocorridos nos períodos de mar baixo (Tortoniano e Pleistoceno), devem ter sido principais fatores que moldaram a atual morfologia da plataforma continental.

O termo Barreiras, utilizado no sentido tradicional, deve ser considerado como Grupo, e não como Formação.

\section{REFERÊNCIAS BIBLIOGRÁFICAS}

ALHEIROS, M. M.; LIMA FILHO, M. A Formação Barreiras. Revisão geológica da faixa sedimentar costeira de Pernambuco, Paraíba e Rio Grande do Norte. Recife: UFPE/ DEGEO, 1991. p. 77-88 (Estudos Geológicos - série B, Estudos e Pesquisas, 10).

ALHEIROS, M. M.; LIMA FILHO, M. F.; MONTEIRO, F. A. J.; OLIVEIRA FILHO, J. S. Sistemas deposicionais na Formação Barreiras no Nordeste Oriental. In: CONGRESSO BRASILEIRO DE GEOLOGIA, 35., 1988, Belém. Resumos... 
Belém: SBG, 1988. p. 63.

ARAI, M. Dinoflagelados (Dinophyceae) miocênicos do Grupo Barreiras do Nordeste do estado do Pará (Brasil). Revista Universidade de Guarulhos, Geociências, ano II (número especial), p. 98-106, 1997.

ARAI, M.; NOVAIS, L. C. C. Microflora de Comandatuba BA: novos reforços para a idade miocênica do Grupo Barreiras. Paleontologia em Destaque: Boletim Informativo da Sociedade Brasileira de Paleontologia, Porto Alegre, ano 21, n. 53, p. 9, 2006.

ARAI, M.; SHIMABUKURO, S. The Tortonian unconformity and its relation with the stratigraphic framework of the Barreiras Group and correlative units (Neogene, Brazil). In: LATINAMERICAN CONGRESS OF SEDIMENTOLOGY, 3., 2003, Belém. Abstracts... Belém: MPEG, 2003. p. 263-264.

ARAI, M.; UESUGUI, N.; ROSSETTI, D. F.; GOES, A. M. Considerações sobre a idade do Grupo Barreiras no Nordeste do Estado do Pará. In: CONGRESSO BRASILEIRO DE GEOLOGIA, 35., 1988. Belém. Anais ... Belém, SBG 1988. v. 2, p. 738-752.

ARAI, M.; TRUCKENBRODT, W.; NOGUEIRA, A. C. R.; GOES, A. M.; ROSSETTI, D. F. Novos dados sobre estratigrafia e ambiente deposicional dos sedimentos Barreiras, NE do Pará. In: SIMPÓSIO DE GEOLOGIADAAMAZÔNIA, 4., 1994, Belém. Boletim de Resumos Expandidos... Belém, SBG, 1994. p. 185-187.

ARAÚJO, V. D.; REYES-PERES, Y. A.; LIMA, R. F.; SANTOS, D. A. S.; PELOSI, A. P. M. R.; CÓRDOBA, V. C.; LIMA FILHO, F. P. Análise faciológica e interpretação paleoambiental de rochas da Formação Barreiras, litoral oriental do Rio Grande do Norte. In: CONGRESSO ABEQUA, 10., 2005, Guarapari. Resumos Expandidos... Guarapari, ABEQUA, 2005. (1 CD-ROM).

BEURLEN, K. Introdução à estratigrafia geral e comparada. Recife: Expansão Gráfica, 1964. 440 p.

BIGARELLA, J. J. The Barreiras Group in Northeastern Brazil. Anais da Academia Brasileira de Ciências, Rio de Janeiro, v. 47 (Suplemento), p. 365-393, 1975.

BOLTOVSKOY, E. Ihering's hypothesis in the light of foraminiferological data. Lethaia, Oslo, v. 24, p. 191-198, 1991.

BURCKLE, L. H.; KEIGWIN, L. D.; OPDYKE, N. D. Middle and Late Miocene stable isotope stratigraphy: correlation to the paleomagnetic reversal record. Micropaleontology, v. 28, n. 4 , p. 329-334, 1982.

COSTA, J. B. S.; BORGES, M. S.; BEMERGUY, R. L.; FERNANDES, J. M. G.; COSTAJr., P. S.; COSTA, M. L. Evolução cenozóica da região de Salinópolis, Nordeste do Estado do Pará. Geociências, São Paulo, v. 12, n. 2, p. 373-396, 1993.

FERRAZ, C. M. L.; VALADÃO, R. C. Barreiras: formação ou grupo? (contribuições da análise geomorfológica do litoral sul da Bahia e das "chapadas" do Jequitinhonha). In: CONGRESSO ABEQUA, 10., 2005, Guarapari. Resumos Expandidos... Guarapari: ABEQUA, 2005. (1 CD-ROM).

HAQ, B. U.; HARDENBOL, J.; VAIL, P. R. The chronology of Fluctuating Sea Level since the Triassic. Science, Washington, v. 235, p. 1156-1167, 1987.

ICS - International Commission on Stratigraphy. Lithostratigraphic units. Chapter 5. Disponível em: $<$ http://www.stratigraphy.org >. Acesso em: 24 jan. 2006.

LEITE,F.P.R.; BERNARDES-DE-OLIVEIRA, M.E.; OLIVEIRA, P. E.; SILVESTRE-CAPELATO, M. S.; ARAI, M.; TRUCKENBRODT, W. Palinofloras miocenas da Formação Pirabas e Grupo Barreiras, na Região Bragantina, estado do Pará, Brasil. Revista Universidade de Guarulhos, Geociências, Guarulhos, ano II (Número Especial), p. 128-140, 1997.

LEITE, F. P. R. Palinofloras neógenas da Formação Pirabas e Grupo Barreiras, área litorânea nordeste do estado do Pará, Brasil. 1997. 102 f. Dissertação (Mestrado) - Instituto de Geociências, Universidade de São Paulo, São Paulo, 1997.

MENDES, J. C.; PETRI, S. Geologia do Brasil. Rio de Janeiro: Instituto Nacional do Livro, 1971. 207 p.

MORAIS, R. M. O.; MELLO, C.L.; COSTA, F. O.; RIBEIRO, C. S. Estudo faciológico de depósitos terciários (formações Barreiras e Rio Doce) aflorantes na porção emersa da Bacia do Espírito Santo e na região emersa adjacente à porção norte da Bacia de Campos. In: CONGRESSO ABEQUA, 10., 2005, Guarapari. Resumos Expandidos... Guarapari: ABEQUA, 2005. (1 CD-ROM).

NETTO, R. G.; ROSSETTI, D. F. Ichnology and salinity fluctuations: a case study from the early Miocene (Lower Barreiras Formation) of São Luís Basin, Maranhão, Brazil. Revista Brasileira de Paleontologia, v. 6, p. 5-18, 2003.

OLIVEIRA, A. I.; LEONARDOS, O. H. Geologia do Brasil. 
Rio de Janeiro: Comissão Brasileira dos Centenários de Portugal, 1940. 472 p., 36 estampas.

OLIVEIRA, A. I.; LEONARDOS, O. H. Geologia do Brasil. Rio de Janeiro: Ministério da Agricultura, Serviço de Informação Agrícola, 1943.813 p., 37 estampas (Série Didática, 2).

PETRI, S. Foraminíferos e o ambiente de deposição dos sedimentos do Mioceno do Recôncavo Baiano. Revista Brasileira de Geociências, v. 2, n.1, p. 51-67, 1972.

RÄSÄNEN, M.E.; LINNA, A. M.; SANTOS, J.C. R.; NEGRI, F. R. Late Miocene Tidal Deposits in the Amazonian Foreland Basin. Science, v. 269, n. 5222, p. 386-390, 1995.

ROSSETTI, D. F. Facies analysis of the Pirabas/Barreiras succession in the northern São Luís Basin: a wave-dominated estuary. In: CONGRESSO ABEQUA, 10., 2005, Guarapari. Resumos Expandidos... Guarapari: ABEQUA, 2005.(1 CD-ROM).

ROSSETTI, D. F. Stratigraphic evolution of Miocene deposits (Pirabas/Barreiras Formation) in northern Brazilian basins. In: CONGRESSO ABEQUA, 10., 2005b, Guarapari. Resumos Expandidos... Guarapari, ABEQUA, 2005. (1 CD-ROM).

ROSSETTI, D. F. The estuarine nature of the Barreiras Formation in the Rio Capim Area, northern Brazil. In: CONGRESSO ABEQUA, 10., 2005c, Guarapari. Resumos Expandidos... Guarapari, ABEQUA, 2005. (1 CD-ROM).

ROSSETTI, D. F.; GÓES, A. M.; TRUCKENBRODT, W. A influência marinha nos sedimentos Barreiras. Boletim do Museu Paraense Emílio Goeldi, série Ciências da Terra, Belém, v. 2, p. 17-29, 1990.

ROSSETTI, D. F.; TRUCKENBRODT, W.; GÓES, A. M. Estudos paleoambiental e estratigráfico dos sedimentos Barreiras e pós-Barreiras na Região Bragantina, Nordeste do Pará. Boletim do Museu Paraense Emílio Goeldi, série Ciências da Terra, Belém, v. 1, p. 25-74, 1989.

SALIM, J.; SOUZA, C. J.; MUNIZ, G. C. B.; LIMA, M. R. Novos subsídios para a elucidação do episódio "Barreiras" no Rio Grande do Norte. In: SIMPÓSIO DE GEOGRAFIA, 7., 1975, Fortaleza. Atas... Fortaleza, 1975, p. 149-158.

SANTOS, P. F.; MELLO, C. L.; MORAIS, R. M. O.; RIBEIRO, C. S. Aplicação do radar de penetração subterrânea na caracterização em subsuperfície de depósitos da Formação Barreiras. In: CONGRESSO ABEQUA, 10., 2005, Guarapari. Resumos Expandidos... Guarapari: ABEQUA, 2005. (1 CD-ROM).
SHIMABUKURO, S.; ARAI, M. A transgressão marinha miocênica no Brasil: considerações baseadas no estudo do Grupo Barreiras e da Formação Pirabas. Anais da Academia Brasileira de Ciências, v. 71, n. 1, p. 144, 1999.

SHIMABUKURO, S.; ARAI, M. The Tortonian unconformity: its relation to the stratigraphic framework of the Barreiras Group (Brazilian coastal Cenozoic deposits). In: INTERNATIONAL GEOLOGICAL CONGRESS, 31., 2000, Rio de Janeiro. Abstracts... Rio de Janeiro: CPRM, 2000. (1 CD-ROM).

SHIMABUKURO, S.; ARAI, M. A Discordância Tortoniana e sua relação com o arcabouço estratigráfico do Grupo Barreiras e unidades correlatas (Neógeno do Brasil). In: CONGRESSOBRASILEIRODEPALEONTOLOGIA, 17., 2001, Rio Branco. Boletim de Resumos... Rio Branco: SBP, 2001, p. 54.

TRUCKENBRODT, W.; NOGUEIRA, A. C. R.; GOES, A. M.; ARAI, M. Conteúdo fossilífero e estruturas sedimentares nos sedimentos Barreiras, NE do Pará. In: CONGRESSO BRASILEIRO DE GEOLOGIA, 38., 1994, Camboriú. Boletim de Resumos Expandidos... Belém: SBG, 1994. v. 3, p. 210-211.

VASCONCELOS, P. Métodos de geocronologia de intemperismo e suas aplicações a eventos de sedimentação continental durante o Neógeno. In: CONGRESSO ABEQUA, 10., 2005. Guarapari, Boletim de Resumos... Guarapari, ABEQUA, 2005, p. 258.

VIANA, A. R.; CASTRO, D.; KOWSMANN, R. O. A discordância do Mioceno médio/superior: um marco regional no talude da bacia de Campos. In: CONGRESSO BRASILEIRO DE GEOLOGIA, 36., 1990, Natal. Anais... Natal: SBG, 1990. v. 1, p. 313-323.

WEBB, S. D. Biological implications of the Middle Miocene Amazon Seaway. Science, v. 269, n. 5222, p. 361-362, 1995. 